\title{
Déterminisme de la répartition de la pigmentation dans le pelage et la peau des Mammifères
}

\author{
J. ROUGEOT \\ I.N.R.A. \\ I.N.R.A., Laboratoire des Pelages, Toisons et Fourrures \\ Centre de Recherches zootechniques, \\ 78350 Jouy-en-Josas
}

\begin{abstract}
Résumé
Chez les mammifères sauvages, la pigmentation du pelage par les mélanines assure deux fonctions, le camouflage et la signalisation (avertissement, menace, reconnaissance, ralliement). Par contre la fonction de protection de la mélanine cutanée contre les radiations (UV) qui est très importante chez les animaux dénudés, et en particulier chez l'Homme, est tout à fait secondaire dans le pelage dont la masse de kératine, constituée par les poils, représente un écran suffisant.

La répartition de mélanines dans le tégument, les phanères et en particulier le pelage, dépend de nombreux facteurs qui sont héréditaires et dont les interactions sont complexes : synthèse des mélanines dans le mélanocyte, migration et évolution des mélanoblastes, interaction du mélanocyte avec le milieu cutané et kératinocytaire. La multiplicité des combinaisons possibles aboutit à une très grande variété de systèmes de répartitions de diverses couleurs dans l'appareil cutané. Ils sont parfaitement définis, d'une part, dans l'espèce, chez les Mammifères sauvages, où ils ont une valeur adaptative et sont déterminés par l'action de la sélection naturelle, d'autre part, dans la race chez les espèces domestiques, où la sélection est guidée par l'intérêt économique ou esthétique.
\end{abstract}

\section{Introduction}

Chez les animaux, le rôle fondamental de la pigmentation externe est la protection contre les prédateurs. Cette protection peut être d'ordre passif en rendant l'animal le moins visible possible dans le milieu où il vit : c'est le camouflage, qui est de loin la fonction la plus générale. Mais cette protection peut revêtir également un aspect actif, les dessins réalisés sur le corps par la pigmentation ayant valeur de signe de menace, d'avertissement, d'alerte.

L'importance du camouflage dans la sélection vis-à-vis des prédateurs a pu être chiffrée par Cotr (1957) chez Gambusia patruelis. Ces poissons maintenus les uns sur fond clair, les autres sur fond foncé, pendant une période suffisante pour qu'ils acquièrent une pigmentation les faisant se confondre avec leur habitat, sont ensuite répartis sur fonds clairs ou foncés. La mortalité par prédation s'élève à $66 \mathrm{p}$. 100 pour les Gambusia sur fond contrasté contre 34 p. 100 pour les témoins sur fond de même type. 
Chez les Mammifères, la pigmentation, due uniquement à la mélanine, est perçue surtout sur le pelage qui recouvre la presque totalité du corps chez la plupart d'entre eux. Ce pelage, dont la composition et la structure sont strictement définies (groupes folliculaires où les follicules pileux sont répartis selon un ordre hiérarchique, 'es follicules primaires fournissant les poils de garde couvrant les fragiles duvets de la fourrure d'isolation thermique produits, eux, par les follicules pileux secondaires), est un véritable organe de protection contre les chocs, les précipitations, les variations de température. Il se renouvèle non seulement pour maintenir son intégralité, mais aussi pour s'adapter aux changements saisonniers du milieu : pelage long et dru de l'hiver, plat et maigre de l'été, établis à la suite de mues réglées par le photopériodisme.

En ce cas, la peau sous-jacente, efficacement protégée, ne possède que peu ou pas de mélanine et l'on peut admettre que la pigmentation du pelage ne remplit qu'une fonction de camouflage, ou de signalisation, remplie par les poils de garde.

Par contre, lorsque la peau est peu couverte de poils ou dénudée, comme c'est le cas notamment chez l'Homme, elle est plus ou moins chargée de mélanine qui semble alors assumer d'autres fonctions :

- écran ou action protectrice contre les U.V. solaires,

- régulation de la production de vitamine $\mathrm{D}$ qui se synthétise à partir de la provitamine D sous l'action des U.V.,

- thermorégulation,

- adaptation aux affections tropicales.

Bien que non exclusives l'une de l'autre, ces fonctions ont pu évoluer entièrement indépendamment (HADLEY, 1972).

Rappelons enfin que la pigmentation des formations kératiniques est fixée de façon définitive étant donné que les granules de mélanine sont inclus dans les cellules kératinisées (avec la réserve que la couleur peut être altérée par les agents du milieu). En effet, les kératinocytes, en phagocytant les extrémités des dendrites des mélanocytes qui les enlacent, ingèrent les mélanosomes élaborés par ces cellules (unité mélanocyto-kératocytaire de FitzPatrick \& BReathnach, 1963).

Par contre, dans les autres tissus et notamment dans le derme, ce sont les mélanocytes mélaninisants qui seuls contiennent la mélanine (mélanophores chez les vertébrés inférieurs). Il existe donc une interaction entre le milieu et le système mélanocytaire qui intervient de façon importante dans la répartition de la pigmentation dans la peau et les phanères : notons, en ce qui concerne les poils, qu'il n'y a jamais de mélanine dans la cuticule, contrairement au cortex et à la moelle, et que l'alternance de bandes à eumélanine et phéomélanine des poils agouti est en relation avec un changement du milieu kératocytaire du bulbe pileux (Poole, 1975).

\section{1. - Le rôle protecteur de la mélanine chez les Mammifères}

\section{1. - Le camouflage}

Dès 1929, Dice remarque l'adaptation de la couleur du pelage sur fond de l'habitat chez un Mammifère du désert (Perognatus ou pocket mouse) : les individus 
vivant sur les zones de sable blanc ont un pelage blanc, tandis que ceux vivant sur les coulées de lave noire possèdent un pelage noir (en dépit donc du rayonnement solaire intense qui n'est pas réfléchi). Plus tard (1947), le même auteur montre l'efficacité de la sélection par les oiseaux de proie nocturnes sur Peromyscus maniculatus dont la couleur contraste avec le fond de l'habitat. Guthrie (1967) observe en Alaska que les brûlis, en rendant le sol plus foncé, favorisent la pullulation de Citellus undulatus protégés par un pelage foncé. L'adaptation de la couleur du pelage au milieu est encore plus perfectionné chez les Mammifères où les mues saisonnières donnent un pelage coloré en été et blanc en hiver (Lièvre variable, Hermine). Chez ces espèces, les mues sont réglées, comme chez tous les Mammifères, par le photopériodisme. Mais Rust (1962) a montré que chez l'Hermine, le remplacement du pelage, induit par le photopériodisme, était d'autant plus rapide que le changement de température était plus brutal.

\section{2. - Le signe de menace ou d'avertissement}

Guthrie \& Petocz (1970) interprètent comme un signe de protection, ayant un sens de menace, les masques pigmentés, souvent fort précis, sur la tête de Mammifères dépourvus d'armes de défense : tâches noires foncées sur la lèvre de certains Cervidés qui auraient la signification d'un renforcement de la canine qui existait chez leurs ancêtres ; tâches blanches prolongeant les canines (Phacochère, Chevrotain) ou tâches blanches et noires sur les oreilles et la face exagérant les dimensions des cornes (Antilopes, Cervidés). Guthrie (1970) pense, dans cet ordre d'idée, que la barbe de l'Homme, en élargissant les joues, exprime la menace, de façon d'autant plus prononcée que les poils sont noirs. D'ailleurs, le noir a, par rapport aux couleurs claires, une signification de menace : mâle plus foncé que les femelles (même chez l'Homme). Mais SEARLe (1968) précise que le signal de menace, ou d'alarme, est blanc chez les animaux nocturnes, tandis qu'il est noir chez les animaux diurnes. Ce signal, normalement caché par la position des oreilles ou de la queue, peut être brusquement dévoilé.

Les signes d'avertissement préviennent quant à eux de l'existence d'un danger réel : on interprète de cette façon l'inversion de la couleur du pelage du Sconce, blanc sur le dos, foncé sur le ventre, qui avertit de la puanteur de son propriétaire.

\section{3. - Comportement}

L'avant du corps foncé (Bovins, Cervidés) serait un signe d'attaque, de menace, tandis que l'arrière du corps plus clair signifie la soumission lorsque l'animal se tourne. L'arrière blanc des Cervidés est considéré comme un signal de ralliement dans la fuite.

Comme autres fonctions visuelles de la pigmentation, on peut citer les tâches des Félins les confondant avec les tâches de la lumière dans le feuillage, les rayures des Zèbres dissimulant les mouvements dans la fuite; dans un autre ordre d'idées, citons enfin les orbites entourés de blanc des prédateurs pour concentrer la lumière et les traits noirs, qui les accompagnent souvent et facilitent la visée. 


\section{2. - Fonctions physiologiques de la mélanine}

\section{1. - Thermorégulation}

La pigmentation n'interviendrait que dans la fonction de réflection ou d'absorption des rayons caloriques du milieu; elle n'aurait aucun rôle dans la déperdition de chaleur (Svinla, 1956; rats blancs et noirs). Cependant, la fonction de réflexion serait toujours subordonnée à celle, prioritaire, du camouflage. A noter que les albinos sont capables de consommer plus d'oxygène à basse température que les individus colorés : il s'agirait d'une liaison génétique des caractères.

En fait, le rôle physiologique de la mélanine apparaît plus nettement lorsque la peau est elle-même pigmentée, ce qu'on trouve chez les Mammifères peu pourvus ou dépourvus de pelage.

\section{2. - Protection contre les U.V.}

(Blum, 1961 ; PathaK, 1967 ; Szabo, 1967)

Chez l'Homme, il existe une corrélation entre la fréquence du cancer de la peau et la couleur de sa peau, selon l'intensité de l'insolation de la région habitée. Aussi, bien que la peau pigmentée par la mélanine favorise l'absorption de calories, la fonction de protection contre les U.V. prévaut chez les races humaines noires. On sait que la mélanine protège les tissus contre les rayons actiniques en tant que radical libre stable acceptant facilement les électrons (GAN et al., 1977). Mais le mécanisme n'est pas encore bien compris et est même discuté (MENon \& HABERMAN, 1977). De toute façon, on constate que chez les mammifères nus ou peu pourvus de poils des régions tropicales (Buffles, Rhinocéros...), la peau est généralement pigmentée, alors qu'elle ne l'est qu'exceptionnellement chez les Mammifères à pelage épais et subissant des mues saisonnières (la peau ne bleuit que lorsque les follicules pileux sont en anagène, la mélanine des follicules pileux étant vue par transparence dans la peau ; exceptionnellement, la peau est tâchetée chez le Cobaye et certaines races de moutons, mais les mues saisonnières n'existent pas chez ces espèces ; quant à la peau de l'ours blanc, elle est totalement pigmentée en noir foncé : est-ce en relation avec la fréquence des mélanomes ?).

\section{3. - Synthèse de la vitamine $D$ (Loomis, 1963)}

En réduisant l'action des U.V., la mélanine ralentit la synthèse de la vitamine D à partir de la provitamine chez les peaux pigmentées normalement ou temporairement (bronzage), évitant ainsi une hypervitaminose dangereuse (?). De fait, dans les pays peu ensoleillés, les immigrants noirs se révèlent très sensibles à la carence en vitamine $\mathrm{D}$, le rayonnement naturel des U.V. étant très faible.

2.4. - Rôle homéostatique de la mélanine en relation avec les affections tropicales (WASSERMANN, 1965)

L'adaptation aux maladies tropicales impliquerait une plus grande activité du système réticulo-endothélial, donc une dépression de l'activité des surrénales et par 
rétroaction, une élévation de la sécrétion de $\mathrm{ACTH}$ et $\mathrm{MSH}$ en relation avec une hyperpigmentation cutanée. Par ailleurs, il existe de la mélanine circulante chez les noirs : cette mélanine, transportée par les leucocytes, exercerait une action de tampon générale sur les phénomènes d'oxydo-réduction résultant d'un métabolisme élevé.

\section{3. - La répartition de la mélanine dans la peau et le pelage}

\section{1. - Répartition de la mélanine dans la peau : cas des Primates}

Dans leur étude exhaustive sur 40 espèces de Primates supérieurs, MACHIDA \& Perkins (1947) ont observé des mélanocytes dans tous les organes ou tissus de la peau, mais jamais partout en même temps et avec une répartition très variable suivant les zones : en général, les mélanocytes mélaninisants sont localisés dans les parties découvertes (paupières, nez, lèvres, oreilles externes, surface de friction) en étant localisés soit dans l'épiderme (unités mélanocyto-kératocytaires), soit dans le derme où leur répartition est très diverse :

- dans les vaisseaux sanguins et les nerfs à myéline ;

- dans le tissu conjonctif lui-même en îlots isolés, entre les follicules pileux ou autour de ceux-ci dans la couche papillaire ou la couche réticulée, ou dans les deux, distribués de façon uniforme ou non;

- dans les follicules pileux, gaine externe et bulbe pileux exclusivement;

- dans les glandes sébacées et les glandes sudoripares eccrines et apocrines.

On doit remarquer qu'à mesure qu'on s'élève dans l'échelle phylogénique chez ces Primates, la pigmentation dans la peau a de plus en plus tendance à être épidermique.

\section{2. - La panachure}

La panachure consiste en la présence de tâches blanches, avec une répartition et un contour souvent bien définis dans un pelage pigmenté. Ces tâches blanches proviennent de l'absence de mélanocytes. L'étude génétique en a été faite de façon très précise chez la Souris, dans la mesure où l'on a pu déterminer chez cette espèce la migration des mélanoblastes à partir de la crête neurale (RAWLES, 1947 ; MAYER, $1967,1977)$ et leur répartition suivant des zones de peau déterminées pour chaque souche de mélanoblastes (MINTZ, 1967). Ces mélanoblastes peuvent être défectueux par eux-mêmes, dès leur constitution dans la crête neurale ou bien rencontrer un milieu hostile auquel ils sont plus ou moins sensibles et qui s'oppose à leur développement ou à leur différenciation en mélanocyte. On distingue donc les schémas suivants (QUEVEDo, 1973) :

- les mélanoblastes défectueux sont incapables de migrer ou de se différencier en mélanocytes : lethal spotting $(l s / l s)$, splotch $(S p / S p$, lethal également), dominant spotting $\left(W^{v} / W^{v}\right.$, viable);

- la différenciation en mélanocytes ne peut s'effectuer, ou bien les mélanocytes 
ne survivent pas en raison de l'incompatibilité avec le milieu : steel $(s l / s l$, la peau est inhibitrice), belted ( $b t / b t$, le follicule pileux est inhibiteur);

- les deux systèmes se combinent : pie $(s / s)$.

\section{Conclusion}

Nos connaissances sur le mécanisme de la répartition de la mélanine dans l'appareil cutané des Mammifères sont donc bien fragmentaires et bien hypothétiques, malgré quelques découvertes brillantes de ces deux dernières décennies, et l'on demeure confondu devant la précision des dessins pigmentés du pelage à laquelle a abouti la sélection, qu'elle soit naturelle ou non.

Reçu pour publication en mars 1981.

\section{Summary}

\section{Determinism of coat and skin pigmentation patterns in Mammals}

The pigmentation of the coat of wild Mammals has two functions : concealing and displaying (alarm, threat, identification, rallying). On the other hand, the function of the skin melanins, protection against radiation (UV), which is of paramount importance in nude mammals, especially man, is quite secondary in hairy animals because the hair keratin bulk itself is an efficient screen.

The pigmentation patterns of the tegument and its appendages, especially in the coat, are conditioned by several hereditary factors : melanocyte melanin synthesis, melanoblast migration and evolution, interaction between the melanocytes and the skin and keratinocyte environments. These factors are interrelated, in many ways, among themselves and to their environment. The multiplicity of putative combinations gives a wide range of colours and of colour patterns in the cutaneous system. These patterns are well defined first of all in wild mammalian species, where they have an adaptative function and are the effects of natural selection and, secondly, in domestic breeds which are selected for economic or aesthetic reasons.

\section{Références bibliographiques}

Blum H.F., 1961. Does the melanin pigment of human skin have adaptative value ? An assay in human ecology and the evolution of race. Quart. Rev. Biol., 36, 50-63.

Cotт HB., 1957. Adaptative colorations in animals. Methuen and Co., London.

Dice L.R., 1929. Description of two new pocket mice and a new woodrat from New Mexico. Occas. Pap. Mus. Zool. Univ. Mich., 203, 1-4.

Dice L.R., 1947. Effectiveness of selection by Owls of Deer Mice (Peromyscus maniculatus) which contrast in color with their background. Contrib. Lab. Vertebrate Biol. Univ. Mich., 34, 1-20.

FitzPatrick T.B., Breathnach A.S., 1963. Das epidermale melanin-Einheit-System. Dermatol. Wochenschr., 147, 481-489. 
FrISCH O. VON, 1973. Animal camouflage. Collins, Franklin Watts.

Gan E.V., Lamb K.M., Haberman H.F., Menon I.A., 1977. Oxidizing and reducing properties of melanin. Br.J. Dermatol., 96, 25-28.

Guthrie R.D., 1967. Fire melanism among mammals. Am. Mid. Nat., 77, 227-230.

Guthrie R.D., 1970. Evolution of human threat display organs. Evol. Biol., 4, 257-300.

Guthrie R.D., Petocz R.G., 1970. Weapon automimicry among Mammals. Am. Nat., 104, $585-588$.

HADLEY M.E., 1972. Functional significance of vertebrate integumental pigmentation. Am. Zool., 12, 63-76.

Loomis W.F., 1967. Skin-pigment regulation of vitamin-D biosynthesis in Man. Science, 157, 501-506.

Machida H., Perkins E.M. Jr., 1967. In Montagna W., Funan Hu, The pigmentary system. Adv. Biol. Skin, 8. The distribution of melanotic melanocytes in the skin of subhuman Primates, 41-58. Pergamon Press, Oxford, New York.

MAYER T.C., 1967. Temporal skin factors influencing the development of melanoblasts in piebald Mice. J. exp. Zool., 166, 397-404.

MAYER T.C., 1970. A comparison of pigment cell development in albino, steel and dominant spotting mutant Mouse embryos. Dev. Biol., 23, 297-309.

Menon I.A., Haberman H.F., 1977. Mechanism of action of melanins. Br. J. Dermatol., 97, $109-112$.

MinTz P., 1967. Gene control of mammalian pigmentary differentiation. 1. - Chorial origin of melanocyts. Proc. nat. Acad. Sci., U.S.A., 58, 344-351.

Pathak M.A., 1967. In Montagna W., Funan Hu, The pigmentary system. Adv. Biol. Skin, 8. Photobiology of melanogenesis : biophysical aspects, 397-420. Pergamon Press, Oxford, New York.

Poole T.W., 1975. Dermal-epidermal interactions and the action of alleles at the agouti locus in the Mouse. Dev. Biol., 42, 203-210.

Quevedo W.C. Jr., 1973. Genetic control of melanin metabolism within the melanin unit of mammalian epidermis. J. invest. Dermatol., 60, 407-417.

Rawles M.E., 1947. Origin of piment cells from the neural crest in the Mouse embryos. Physiol. Zool., 20, 248-266.

Rust C.C., 1962. Temperature as a modifying factor in the spring pelage change of shorttailed Weasels. J. Mammal., 43, 323-328.

SeArle A.G., 1968. Comparative genetics of coat colour in Mammals. Logos Press and Academic Press, London and New York.

SviHLA A., 1956. The relation of coloration in mammals to low temperature. J. Mammal., 37, 378-381.

Szabo G., 1967. In Montagna W., Funan Hu, The pigmentary system. Adv. Biol. Skin, 8. Photobiology of melanogenesis : cytological aspects with special reference to differences in racial coloration, 379-396. Pergamon Press, Oxford, New York.

Wassermann H.P., 1965. Human pigmentation and adaptation. Arch. Environ. Health, 11, 691-694. 